DOI: $10.35643 /$ Info.25.2.4

Artículo original

\title{
Las bibliotecas en la sociedad de consumo: estudio de caso de las bibliotecas de la Universidad de la República (Uruguay)
}

\author{
Libraries in the Consumer Society: case study of the Libraries of the \\ University of the Republic (Uruguay) \\ Bibliotecas na sociedade de consumo: estudo de caso das bibliotecas da \\ Universidade da República (Uruguai)
}

\begin{abstract}
Silvana Mariel González Carballal ${ }^{\mathrm{a}}$
${ }^{a}$ Jefa de Hemeroteca del Departamento de Documentación y Biblioteca (Facultad de Humanidades y Ciencias de la Educación, Universidad de la República). Correo electrónico: sgz@adinet.com.uy. ORCID: 0000-0001-7525-9998.
\end{abstract}

\section{Resumen}

Se presenta un estudio realizado a fin de obtener el título de Magíster en Información y Comunicación de la Facultad de Información y Comunicación (FIC) de la Universidad de la República (Udelar). Este plantea que las bibliotecas y su vínculo actual con los usuarios son producto del modelo social contemporáneo, influenciado por características de la cultura de consumo que le son propias, entre las que la tecnología cobra especial relevancia. Se exploró su uso e imagen actual, su relación con los usuarios, conductas de lectura y búsqueda de información. Para ello, se aplicó una encuesta en línea, entre julio y agosto del 2017, dirigida a estudiantes de grado de la Universidad de la República. Se observó que se asocian mayormente con libros, y que, aunque se consideran importantes, su uso suele ser esporádico y son más utilizadas como espacio físico. Si bien se modifica la ergonomía de la lectura, adquieren una función de último recurso para acceder a material no disponible por otros medios, dejando de ser la única fuente de información y el único centro autorizado de lectura; las búsquedas de información comienzan mayormente a través de Google. Aunque se reconocen más confiables y con información de mejor calidad, Internet es más adecuada a los estilos de vida. Por lo tanto, se estima la hipótesis confirmada.

Palabras clave: BIBLIOTECAS UNIVERSITARIAS; CONSUMO; USUARIOS; INTERNET; LECTURA.

\begin{abstract}
A study carried out in order to obtain the title of Master in Information and Communication (FIC, Udelar) is presented. It states that libraries and their current link with users is the product of the contemporary social model, influenced by characteristics of the consumer culture that are its own, with technology taking on
\end{abstract}


special relevance. Its current use and image, its relationship with users, reading behaviors and information search were explored. An online survey was applied, between July and August 2017, aimed at degree students from the University of the Republic. It was observed that libraries are mostly associated with "books", and that, although they are considered important, their use is usually sporadic, they are more used as «physical space». Although the ergonomics of reading are modified, they acquire a function of "last resort" to access material not available by other means, ceasing to be the only source of information and authorized reading center, starting mostly information searches through Google. Although they are recognized as more reliable and with better quality information, the Internet is more appropriate to lifestyles. Therefore, the confirmed hypothesis is estimated.

\section{Keywords: UNIVERSITY LIBRARIES; CONSUMPTION; USERS; INTERNET; READING.}

\section{Resumo}

É apresentado um estudo realizado para a obtenção do título de Mestre em Informação e Comunicação pela Faculdade de Informação e Comunicação (FIC) da Universidade da República (Udelar). Isso sugere que as bibliotecas e seu vínculo atual com os usuários são produto do modelo social contemporâneo, influenciado por características próprias da cultura de consumo, entre as quais a tecnologia assume especial relevância. Foram explorados seu uso e imagem atuais, sua relação com os usuários, comportamentos de leitura e busca de informação. Para tanto, foi aplicada uma pesquisa online, entre julho e agosto de 2017, destinada a alunos de graduação da Universidade da República. Observouse que eles estão principalmente associados aos livros e que, embora sejam considerados importantes, seu uso costuma ser esporádico e são mais utilizados como espaço físico. Embora a ergonomia da leitura seja modificada, elas adquirem uma função de último recurso para acessar materiais não disponíveis por outros meios, deixando de ser a única fonte de informação e o único centro de leitura autorizado; as pesquisas de informações começam principalmente no Google. Embora sejam reconhecidas como mais confiáveis e com informações de melhor qualidade, a Internet é mais adequada aos estilos de vida. Portanto, a hipótese confirmada é estimada.

Palavras-chave: BIBLIOTECAS UNIVERSITÁRIAS; CONSUMO; USUÁRIOS; INTERNET; LEITURA.

$\begin{array}{ll}\text { Fecha de recibido: } & \text { 19/07/2020 } \\ \text { Fecha de aceptado: } & 02 / 09 / 2020\end{array}$

\section{Introducción}

En el año 2003, la Online Computer Library Center (OCLC) realizó un estudio que observaba con preocupación que las bibliotecas estaban perdiendo su definición y razón de ser en el contexto de la sociedad de la información y el conocimiento: 
Se ha vuelto cada vez más difícil caracterizar y describir el propósito y la experiencia de usar bibliotecas y otras organizaciones aliadas. El relacionamiento entre el profesional de la información, el usuario y el contenido han cambiado y seguirán cambiando (De Rosa, Dempsey y Wilson, 2004, p. 2).

Partiendo de esta situación, posteriormente se realizaron dos encuestas (De Rosa et al., 2005; 2011), a fin de conocer el porqué de lo mencionado. Se obtuvo como resultado que las bibliotecas estaban cayendo en desuso frente a Internet, su imagen había quedado estancada en la idea de libro y, si bien la información por ellas brindada era considerada confiable y pertinente, su uso no se adaptaba a los estilos de vida de aquel entonces, mientras que Internet, sí.

En el presente, el ritmo de vida suele ser muy agitado: la familia, el trabajo, los desplazamientos, la casa, el estudio, la vida social; el tiempo se torna tirano y escaso. Esta forma de vida acelerada trajo aparejados dispositivos y medios que ponen la información al alcance de la mano.

En esta situación, se considera que se ha producido un cambio en los usuarios que generó un impacto en las bibliotecas. Sobre la base de este nuevo contexto, usan, evalúan, necesitan y demandan información, así como juzgan y prefieren servicios y productos, al igual que entornos edilicios y virtuales, por lo cual, se estima que el estilo de vida determina el uso de información de las personas, entendiéndose que en las bibliotecas existe algo más allá de lo que habitualmente se considera a la hora de evaluarlas. Sobre la base de lo expuesto, se percibe que, en el contexto actual, la conducta de los usuarios de información no solo se ve influenciada por su estilo de vida, sino que existen otros componentes que la integran, tal como el consumo: la conducta de consumo. Lo que se observa en el ejercicio profesional es una brecha entre el comportamiento de los usuarios y el «respeto debido» hacia una institución. Y la pregunta, como dice Pignet (2005), es: ¿deberían los usuarios comportarse de otra manera? Por este motivo se planteó la investigación realizada, a fin de obtener el título de Magíster en Información y Comunicación, mediante el Programa de Desarrollo Académico de la Información y la Comunicación (PRODIC, FIC, Udelar). En ella se analizó la percepción, uso social y expectativas de los usuarios de bibliotecas universitarias en el contexto actual. Los antecedentes que se consiguieron no fueron muchos ni tratan el tema directamente, por lo que se comprueba que el tema en sí no ha sido estudiado ${ }^{1}$. 


\section{Las bibliotecas en el marco social actual}

Como se mencionó antes, al ir construyendo el objeto de estudio, se encontró que eran varios los aspectos a considerar en la situación actual del uso de bibliotecas y pudo establecerse que estas y su relación con los usuarios se encuentran enmarcadas dentro de una sinergia entre un macrocontexto y un microcontexto.

\subsection{Macrocontexto}

Dentro del macrocontexto está la sociedad de la información y el conocimiento, que pone de relieve la importancia de la información en la sociedad actual, su volumen, velocidad y alcance; se desarrolla, mayormente, por el uso de las tecnologías de la información y la comunicación, pero genera, a su vez, una lógica de mercado. También se encuentra la «modernidad líquida» (Bauman, 2015), la cual imprime una impronta particular a esta época, rompiendo las barreras del espacio-tiempo, dándose la mano con la globalización. El tercer factor es la sociedad de consumo, entendido el consumo como parte de la condición humana (Barbosa, 2004; Baudrillard, 2009; Sassatelli, 2012). Aunque, hoy en día, más que en una sociedad de consumo se vive en una cultura de consumo, donde todo objeto se transforma en objeto de mercado y, a su vez, en signo (Baudrillard, 2009). No obstante, la lógica de consumo excede la lógica de trabajo y de los productos materiales, alcanzando otras áreas, tales como cultura, las relaciones humanas y las pulsiones individuales. Todo se transforma en un modelo consumible, con la estetización y el confort a la orden del día.

\subsection{Microcontexto}

En interacción con el anterior, se encuentra el microcontexto, integrado por el mercado de la información, que se ha visto muy modificado con Internet. En él, la información pasa a ser vista como bien público o privado (García Pérez, 2011), y las son bibliotecas mediadoras entre la oferta y la demanda de información. También lo componen las industrias culturales —que las bibliotecas integran las cuales no tienen por qué tener una exclusiva finalidad de lucro. En ellas, los bienes culturales son considerados mercancía, pero adquieren una lógica mercantil de características propias: el valor de cambio — precio — es diferente al de uso, lo 
que puede implicar valoraciones funcionales (placer, ocio, diversión, aceptación, etcétera), emocionales (vivencia, recuerdo, experiencia) o de distinción social (Stolovich, 2001). Se valora más la experiencia personal, dejando de verse a la cultura tanto como una fuente de educación, sino, sobre todo, como una fuente de entretenimiento.

\subsection{Usuarios y bibliotecas}

En el centro de ambos contextos están los usuarios como tales y su relación con las bibliotecas. Puede afirmarse que, actualmente, los usuarios en su conducta y hábitos presentan determinadas características que permiten asimilarlos más a consumidores (Sassatelli, 2012; Lipovetsky y Serroy, 2015). Cabe destacar que no se trata de un tipo de consumo, sino de varios: hay tantos tipos de consumo como consumidores existen. Para ellos pesa más el servicio que el producto y son sumamente experienciales y nómades. Presentan hábitos consumistas y hacen un uso consumista de las bibliotecas (Pignet, 2005). Por otro lado, las bibliotecas de por sí poseen características particulares. Como instituciones que son, enfrentan una crisis global (Domènech, Tirado, Traveset y Vitores, 1999) en la cual, si bien aún su función social permanece intacta, deben adaptarse al modelo social actual - muy diferente al que les dio origen- y al complejo productivo al que pertenecen. Su hegemonía ha sido rota por la tecnología y cobraron gran relevancia sus servicios, más que sus productos, dado que estos se juzgan al instante. Se considera, entonces, que es factible analizar las bibliotecas desde tres perspectivas diferentes: como servicio o producto, en relación con la lectura y como espacio físico. Como servicio-producto, son tanto mediadoras como objetos de consumo de un bien como es la información, donde Internet y el mercado editorial juegan un rol importante. A su vez, se encuentran en medio de una demanda exponencial de información y de nuevos hábitos de su consumo. En cuanto al servicio, cobra vital importancia el rol del bibliotecólogo como formador de la experiencia del usuario, teniendo en cuenta, además, que todo producto no lo es en sí mismo, sino que implica un servicio personal (Baudrillard, 2009). En relación con la lectura, los cambios que ha sufrido el acto de leer marcan una impronta difícil de eludir: se integran las pantallas, cambia la ergonomía, las competencias lectoras y se acentúa su carácter social. No obstante, 
se sigue considerando a la lectura como tal, aquella que se hace en papel. Lo demás adquiere otros nombres: textear, mensajear, chatear, etcétera. (García Canclini et al., 2015). Finalmente, como espacio físico, su uso se ha transformado con los años, ya que las distintas generaciones utilizan los espacios de forma diferente y se marcan las tendencias arquitectónicas modernas con una inclinación hacia el confort y la escenografización de los espacios (Las bibliotecas japonesas..., 2017).

\section{Hipótesis y objetivos}

El estudio consideró que las bibliotecas se encuentran influenciadas en el presente por la cultura de consumo, potenciada por el rol de la tecnología y, en particular, de Internet. El objetivo principal del trabajo fue estudiar los factores mencionados que afectan su imagen, sus usos y la relación biblioteca-usuario, tratando de definir las características de estos últimos, así como, también, cómo influyen en la actualidad las nuevas prácticas de lectura y búsqueda de información.

\section{Metodología}

Se desarrolló una encuesta cuantitativa, con algunas preguntas de carácter cualitativo. Se tomó como universo de estudio a todos los estudiantes de grado de la Universidad de la República (Uruguay), delimitándose cuotas por área: Tecnologías y Ciencias de la Naturaleza y el Hábitat; Ciencias Sociales y Artísticas, y Ciencias de la Salud. Se superó ampliamente el límite mínimo estipulado. La encuesta se aplicó en línea entre los meses de julio y agosto del 2017, a través de la herramienta Google Formularios, y fue difundida por medio de las propias bibliotecas universitarias y los centros de estudiantes.

\section{Resultados}

Se obtuvo un total de 783 respuestas, en su mayoría de mujeres (76\% del total de encuestados) de entre 18 y 20 años (85\% del total de encuestados), que estaban cursando entre el segundo y el penúltimo año de la carrera (46\% del total de encuestados), del área de Ciencias de la Salud (47,6 \% del total de encuestados). 
Gráfico 1: Importancia de las bibliotecas

¿En qué medida considera importante a las bibliotecas en el contexto actual?

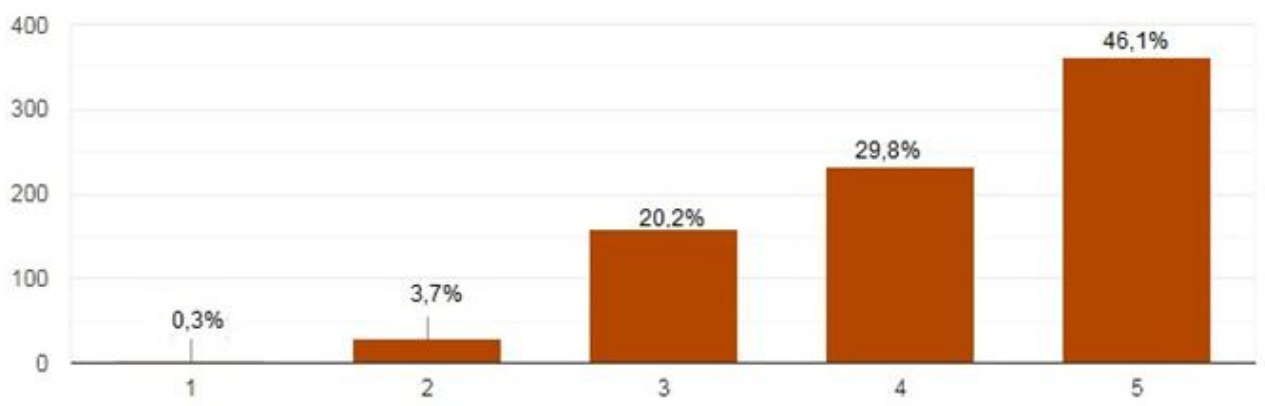

Fuente: González Carballal, S. M. (2019)

En busca de que la primera respuesta fuera la más intuitiva, al inicio del formulario se preguntó: «¿Qué es lo primero que viene a su mente cuando piensa en una biblioteca?». La mayoría de las menciones fueron sobre la palabra libros (43\% de los encuestados), de la que, junto con los términos más frecuentes estudio (13,3\% de menciones) y tranquilidad (6,8\% de menciones)-, se desprende una visión tradicional de las bibliotecas, reafirmándose su vínculo con una forma de lectura específica, con lo físico y con una imagen institucional determinada.

Al medir la relación importancia-asiduidad (véanse Gráficos 1 y 2), se observó que si bien las bibliotecas continúan siendo consideradas muy importantes (46,1 \% del total de encuestados), la asiduidad es mayormente esporádica (65\% del total de encuestados), lo que puede explicarse por las diferentes formas que existen de acceder a la información hoy en día. 
Gráfico 2: Asiduidad

¿Con qué regularidad asiste a las bibliotecas de la Universidad?

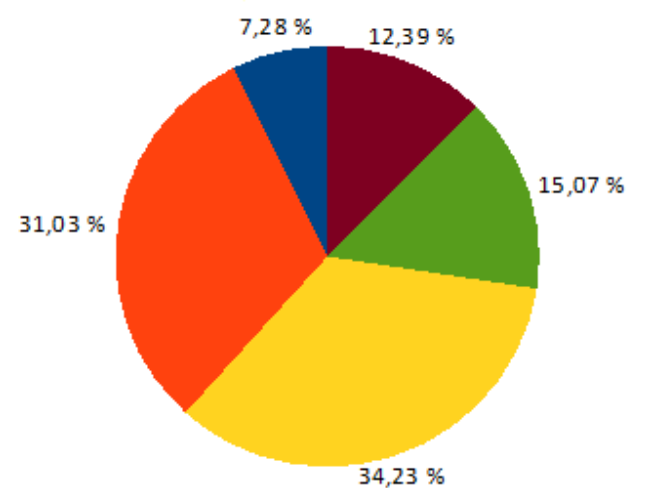

Nunca

- Alguna vez la he usado

- Varias veces al año

- Varias veces al mes

- Varias veces a la semana

Fuente: González Carballal, S. M. (2019)

Sin embargo, se dice que la mayor razón para concurrir es estudiar o trabajar en grupo (26,2 \% del total de encuestados), destacándose el uso de su espacio físico (el 36,7\% de los encuestados la usó para estudiar o trabajar en grupo por el silencio existente, la infraestructura, el gusto por el ambiente, el confort, etcétera). Cobraron relevancia no solo las colecciones, sino también su confort e instalaciones, así como el espacio de socialización que representan. Por otro lado, se afirma que esa asiduidad aumentaría si las bibliotecas fuesen más cómodas y se tuviera más tiempo disponible para asistir a ellas. Del mismo modo, resulta notorio su uso cuando no se consigue el material por otros medios, siendo este un nuevo perfil que adquieren las bibliotecas, el de «respaldo»: «Si no está ahí, no está en ningún lado». Observando en conjunto satisfacción y asiduidad (véase Gráfico 3), si bien una amplia mayoría dijo estar satisfecha con las bibliotecas (67\% del total de encuestados), al comparar esa variable con la frecuencia de uso no se observa una correspondencia directa entre ambas. Esto indicaría que la satisfacción puede estar signada, en algunos casos, más por una imagen social de las bibliotecas que por un uso real de estas, distinguiéndose su institucionalidad y valor social. 
Gráfico 3: Satisfacción vs. Asiduidad

Índice de satisfacción vs. Frecuencia de uso

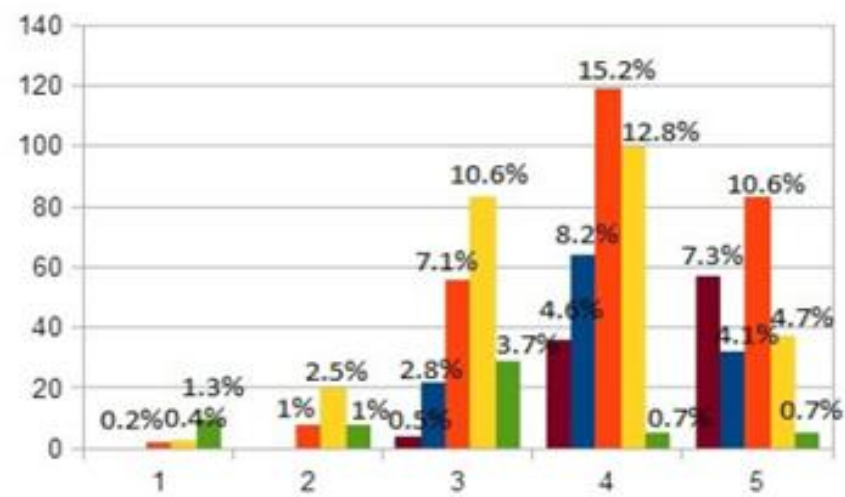

- Varias veces a la semana

- Varias veces al mes

- Varias veces al año

In Alguna vez he ido

- Nunca

Fuente: González Carballal, S. M. (2019)

Se destaca que, en general, no se utilizan otras bibliotecas que no sean las universitarias (76\% del total de encuestados). Por lo que, tal vez, como señala Torres Santo Domingo (2005), estas sean la única puerta de entrada hacia su uso, de allí la importancia de la extensión en este tipo de bibliotecas y del cuidado en la experiencia proporcionada al usuario, que no depende solo de lo brindado, sino también de la construcción personal del individuo.

Al presentar diferentes productos y servicios que se brindan generalmente en bibliotecas universitarias y preguntar por su frecuencia de uso, se observó que cuanto más tradicionales, más utilizados y conocidos eran (véase Gráfico 4). Por el contrario, cuanto más nuevos eran, menos uso y conocimiento se tenía de ellos. Lo dicho revela la dificultad de las bibliotecas para llegar a su público y darse a conocer, lo que reafirma la institucionalización reflejada en el término libro, al describirlas. 
Gráfico 4: Frecuencia de uso según actividad ¿Con qué frecuencia utiliza la biblioteca para las siguientes actividades?

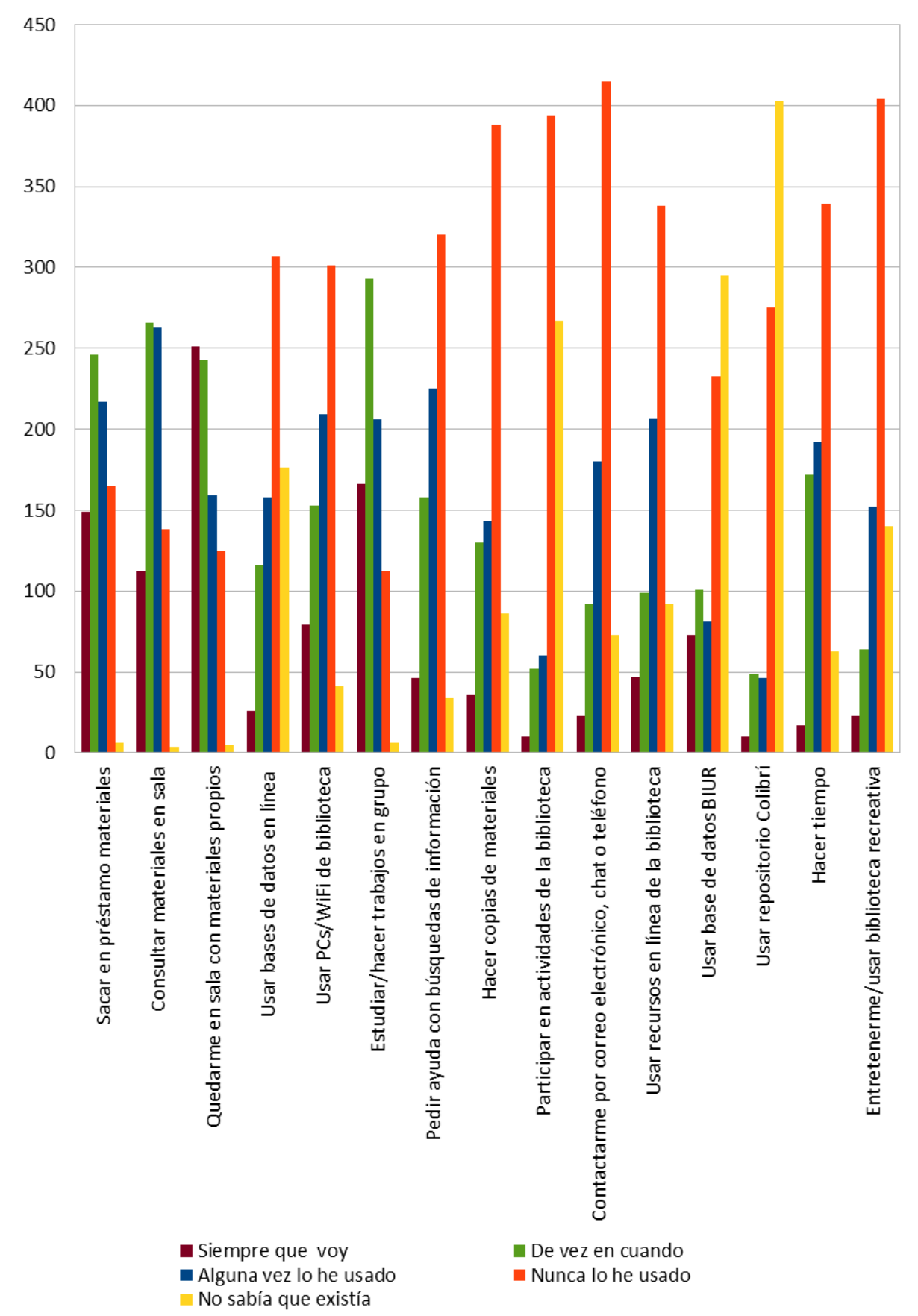

Fuente: González Carballal, S. M. (2019)

Pensando en la estetización y escenografización de los espacios, se ofrecieron a los encuestados dos fotografías de diferentes tipos de bibliotecas, a fin de que eligieran una (véase Imagen 1). La mayoría eligió la segunda foto (86\% de los encuestados), destacando el confort sobre la estética. 
Imagen 1: Tipos de bibliotecas

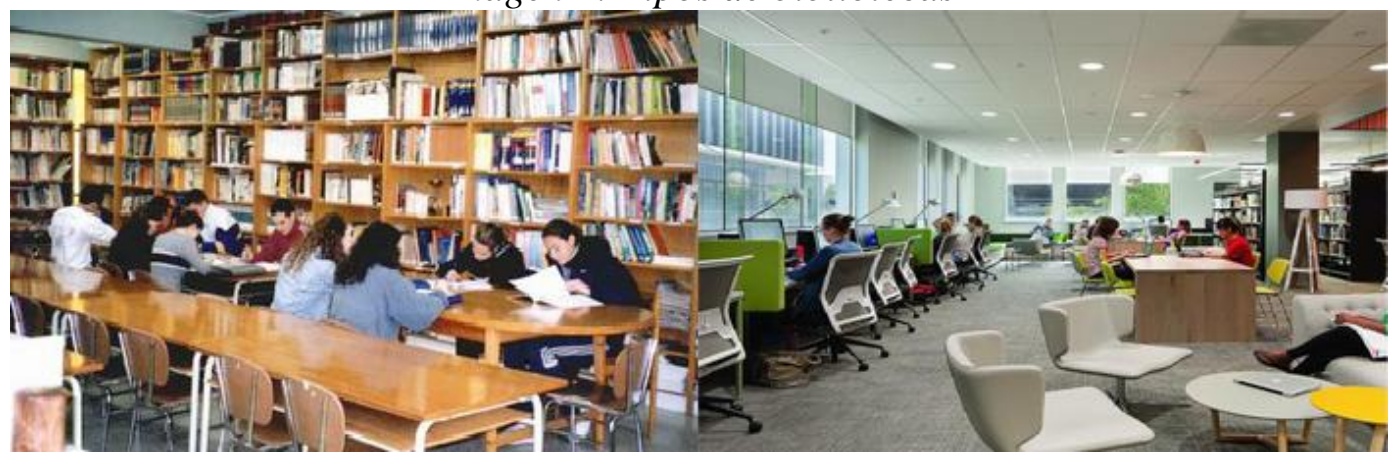

Fuente: González Carballal, S. M. (2019)

Cuando se les solicitó que sugirieran en qué mejorarían las bibliotecas (véase Gráfico 5), el primer lugar lo obtuvo la actualización de las colecciones (74,2 \% de los encuestados), el segundo, la comodidad física y estética del local (59,3\% del total de encuestados), confirmando la importancia de estos aspectos.

\section{Gráfico 5: Mejora de bibliotecas}

¿En qué aspectos usted sugeriría mejorar las bibliotecas?

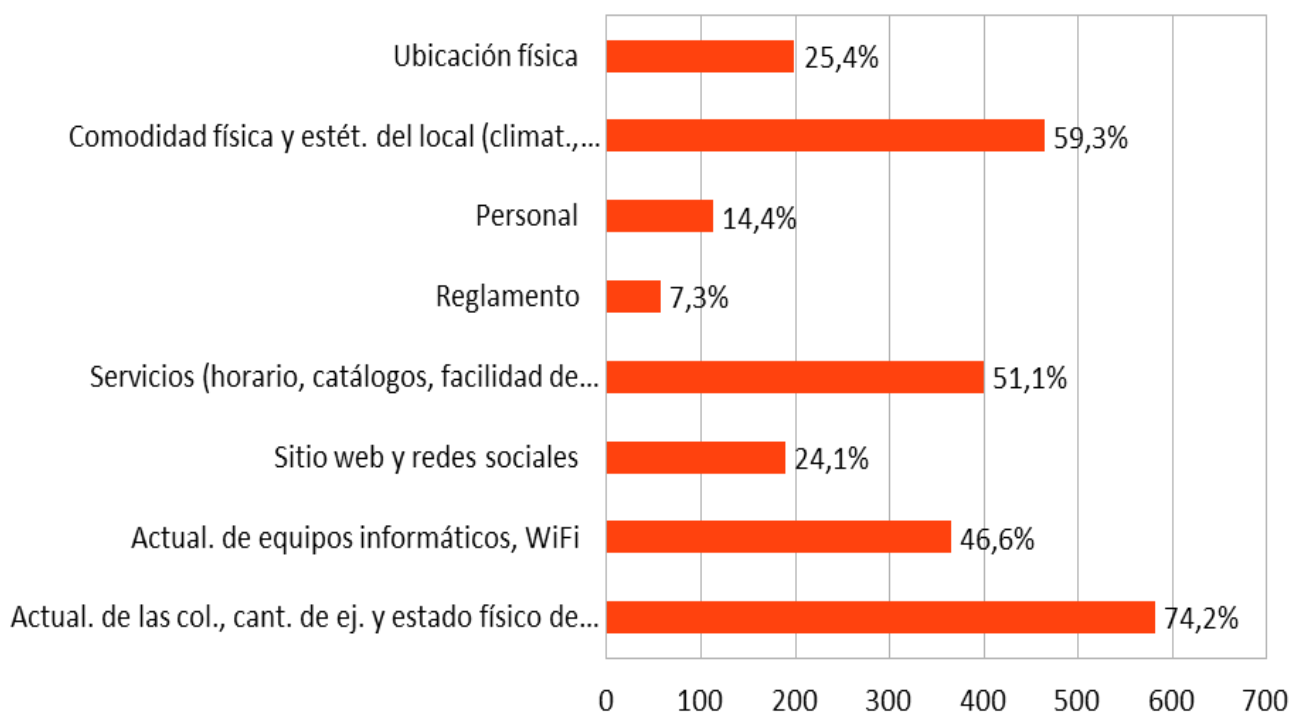

Fuente: González Carballal, S. M. (2019)

En cuanto a la búsqueda de información, una amplia mayoría manifestó comenzarla a través de Google (81,5\% del total de encuestados), y se observa una marcada tendencia hacia la adecuación al estilo de vida y comodidad, con mayor uso de aquello que reviste un fácil acceso, más allá de su calidad o pertinencia. Este resultado se condice con el obtenido en relación con la asiduidad, donde la información digital u otras formas de acceso a la información son uno de los 
grandes responsables de la merma en el uso de las bibliotecas. Al pedirles que las comparen con Internet (véase Gráfico 6), es notorio que las bibliotecas son consideradas más confiables y de mejor calidad (97,1\% del total de encuestados). Sin embargo, Internet destaca en las demás categorías: está más disponible (88,4\% del total de encuestados), es más económico (64\% del total de encuestados), es fácil de usar (79,7\% del total de encuestados), el acceso es inmediato $(94,9 \%$ del total de encuestados), es entretenido $(76,4 \%$ del total de encuestados) y cómodo (80,7 \% del total de encuestados).

Gráfico 6: Biblioteca vs. Internet

Si compara una biblioteca con Internet

¿cuál de los siguientes ítems se aplica a uno de ellos?

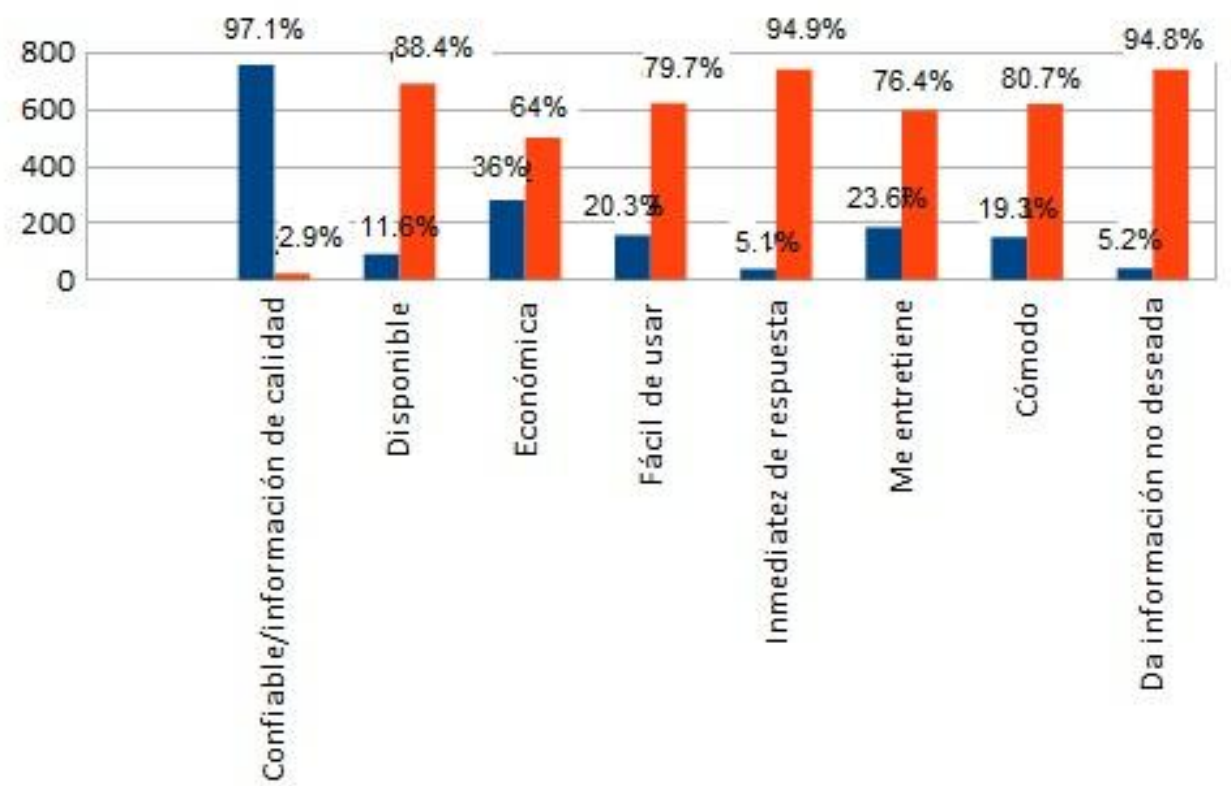

Lo observado puede explicarse a través de lo expuesto por Machlup (citado por Mattelart y Piemme, 1982), quien distingue cinco tipos de conocimientoinformación: el práctico (útil para el trabajo y la toma de decisiones), el intelectual (para la enseñanza científica y cultura general), el pasatiempo o entretenimiento, el espiritual (vinculado a la religión o a las creencias) y el no deseado (adquirido por azar y apenas recordado: ruido). Internet cubre los cinco tipos, incluso al mismo tiempo, y más allá de la necesidad de información o de conocimiento que tenga el individuo, mientras que las bibliotecas solo los abarcan en forma parcial 
entre sí, de acuerdo con los intereses de información o conocimiento del momento.

Lo mencionado se comprueba cuando, ante la solicitud de comparar las bibliotecas con Internet, específicamente en cuanto a satisfacción, adecuación al estilo de vida y necesidades de información (véase Gráfico 7), se obtuvo que ambos son igual de satisfactorios. Sin embargo, Internet posee un mayor número de respuestas «se adapta perfectamente a mi estilo de vida» $(52,1 \%$ del total de encuestados). Esto seguramente se deba a la ubicuidad de esta herramienta y su facilidad de uso.

\section{Gráfico 7: Adaptación a estilo de vida}

Pensando en cada fuente de información, sus necesidades de información y estilo de vida

¿cómo considera ud que se adapta cada una a su estilo de vida?

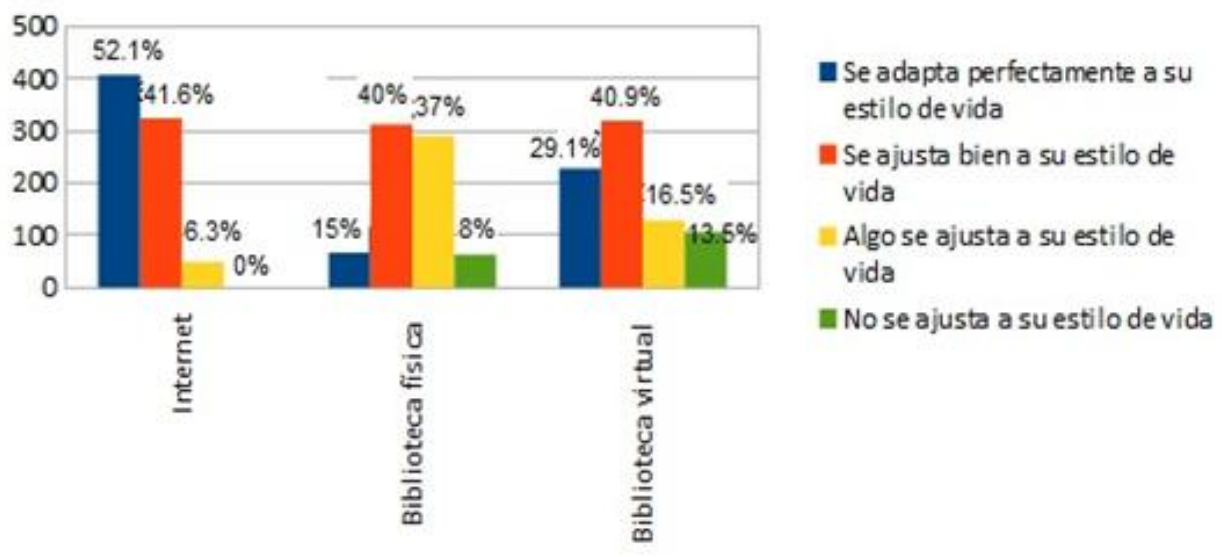

Fuente: González Carballal, S. M. (2019)

Lo visto denota el conflicto existente entre la imagen institucional de las bibliotecas y la sociedad actual. Si bien estas continúan teniendo una alta valoración social, su propia naturaleza y su medio de competencia llevan a que no se ajusten completamente al estilo de vida de las personas, quedando relegadas a otro plano y a otro perfil.

Respecto al uso de dispositivos y lugares habituales de conexión a Internet, se observó que priman los portátiles (teléfono celular y laptops: 97,2 \% y $84 \%$ del total de encuestados, respectivamente), muy acorde a la modernidad líquida en que vivimos (Bauman, 2015). Entre los lugares para conectarse se destacan la casa $(99,2 \%$ de los encuestados) y la facultad $(73,4 \%$ de los encuestados). No 
obstante, se buscan sitios de buena conexión, preferiblemente gratuita, lo que constituye una gran oportunidad para las bibliotecas.

Al consultar sobre sus hábitos de lectura, se confirmó lo observado en el marco teórico: la mayoría prefiere seguir leyendo en papel $(97,6 \%$ del total de encuestados), en su propia casa (98,2\% del total de encuestados), en silencio (61,5\% del total de encuestados), sin día ni horario específico (57,1\% del total de encuestados). Se observa que, como señalan García Canclini et al. (2015), habría que preguntarse primero qué se entiende por lectura, ya que en una época tan tecnológica esta se sigue asociando a la práctica más tradicional. Estas respuestas hablan sobre el tipo de literacidad aún enseñada y aprendida y su correspondencia con la imagen de las bibliotecas que tienen las personas. Sobre la base de los datos obtenidos se establecen las conclusiones y recomendaciones que se presentan a continuación.

\section{Conclusiones}

Se hizo manifiesto que la variabilidad de factores estudiados sí afecta a las bibliotecas, además de la tecnología, que adquiere un rol clave, por lo que se considera la hipótesis confirmada. Al estudiar su entorno, se sugiere incorporar estos aspectos importantes para la sociedad actual. Asimismo, se confirma lo expuesto por los informes de la OCLC mencionados en el marco teórico (De Rosa, Dempsey y Wilson, 2004; De Rosa et al., 2005; De Rosa et al., 2011), observándose que las tendencias señaladas se mantuvieron o se incrementaron con el paso de los años.

En el análisis de los resultados, junto con una conducta de cultura de consumo, lo que más se destacó fue el tema «espacio-tiempo», señalado por Bauman (2015), dado que, constantemente, se declaraba que el tiempo personal, su concordancia o no con el horario de apertura y el desplazamiento eran determinantes en el uso de las bibliotecas. Por lo cual, el estilo de vida se transforma en un factor fundamental.

Es curioso, como fue expuesto, que las bibliotecas mantengan altos índices de satisfacción no reflejados en su frecuencia de uso. Se considera que se establece un doble juego en el que, por un lado, esa satisfacción se encuentra sostenida —en parte- por su rol institucional, su función de bien público y por la enseñanza 
gutenberiana o librocentrista, aún impartida en la educación. Por otro lado, esos mismos factores podrían ser una de las causas que no le permiten evolucionar de su imagen de «libro». Se destaca el nuevo rol de «respaldo» asignado a las bibliotecas: los usuarios las consultan cuando lo que buscan no es encontrado por otros medios, lo que marca un alto índice de confianza y les da un lugar de legitimación informacional y credibilidad institucional.

En cuanto a su imagen, es clara su relación directa con el libro, el estudio y el silencio. No obstante, en las respuestas se constata la necesidad de modernizarlas en distintos aspectos como la estética, el confort, la conexión, la digitalización y las colecciones. Se percibe la idea de querer resignificarlas sin romper su esencia. Se observa que, en opinión de los encuestados, las bibliotecas quedaron en un lugar de no innovación, donde lo que se renueva no alcanza para modificar su perfil. Lo dicho se constata en que, cuanto más habitual y conocido es un producto y servicio, más se usa, mientras que, a la inversa, cuanto más nuevo y diferente era, más se desconoce.

Siguiendo el pensamiento de Seibel (2005) respecto a que el nuevo modelo de comportamiento del consumidor enfatiza la búsqueda de la diversidad y la renovación rápida, la diferenciación no está dada por los productos, sino por los servicios prestados, que se tornan críticos para ganar y retener a un público, cada vez menos afecto a la fidelidad de la oferta.

Es claro que las bibliotecas ya no son la primera ni la única opción para la búsqueda de información. Sin embargo, a la hora de leer, si bien los encuestados realizan numerosas menciones oponiendo Internet al papel, no se observa una disociación, sino una integración de soportes.

Respecto a la lectura en sí, como ya se mencionó, leer, propiamente dicho, es considerado en soporte impreso, lo demás adquiere otros nombres. A su vez, los resultados obtenidos reafirman lo observado por García Canclini (2008) sobre la descontracción en la práctica de la lectura. Se concuerda con el autor en que esta también puede ser una razón por la cual las bibliotecas sean resistidas actualmente y no se justifique incluso, a veces, el desplazamiento hacia ellas. Además, la relación biblioteca-usuario se ve claramente modificada por el uso de Internet, sobre todo en lo que implica el «espacio-tiempo» señalado por Bauman (2015). En los resultados se observó, constantemente, ya sea a favor o en contra, la 
comparación de las bibliotecas con la red global. No obstante la merma en su uso, se destaca la relevancia tomada por su espacio físico como centro de encuentro, de socialización y como punto de conexión multimedial, siendo más valorado por los usuarios el confort que la estetización.

La relación biblioteca-usuario adquiere un nuevo perfil que, más allá de la información, viene de la mano de las características de estos últimos, de un mayor perfil individualista propio de los tiempos modernos. Se coincide con la reflexión de Pignet (2005) sobre que estas prácticas deberían ser tomadas en cuenta en el proyecto social y colectivo de las bibliotecas, invitándolas a repensarse en este sentido. También se concuerda con García Canclini (2008) en que los lectores ya no son tales, sino que sería más apropiado hablar de consumidores o audiencias, en medio de un ambiente de abundancia informacional multimedial, donde las bibliotecas evalúan a los usuarios tanto como los usuarios evalúan a las bibliotecas. Actualmente, se debería pensar en usuarios nómades o esporádicos, que, según los resultados de la encuesta, parecen ser la mayoría. Esto alerta a las bibliotecas a considerar sus necesidades más esporádicas y puntuales, ya que no están listos para aprender sus prácticas, sino que buscarían que ellas se adapten al ritmo de sus necesidades, tal como señala Calenge (2003). O tal vez, como dice Bauman (2015), sería interesante generar productos y servicios sectorizados y exclusivos, ya que la propuesta universalista de las bibliotecas podría estar jugando en contra de las preferencias reales de los usuarios.

Cabe mencionar que, en el total de respuestas obtenidas en la encuesta, no hubo prácticamente diferencias de opiniones entre carreras, edades o géneros. Todos presentaron el mismo perfil característico contemporáneo signado por la liquidez del espacio, el tiempo y la cultura de consumo.

Como se mencionó en el marco teórico, en la actualidad, los bienes y servicios están dotados de simbologías. Por lo tanto, sería bueno que quienes se encuentran a cargo de bibliotecas tengan presente que, además de gestores, son fabricantes de símbolos y significados. Un bibliotecólogo que sabe sacar partido a los recursos con que cuenta y le hace vivir una buena experiencia al usuario es el activo más importante de la biblioteca. Según la opinión de Torres Santo Domingo (2005), se debe volver a dotar de sentido la palabra biblioteca, teniendo en cuenta su futuro en función de cuatro escenarios posibles: cambiar, desaparecer, resignificarse o 
continuar como están. Queda en manos de los profesionales de la información la responsabilidad del cambio que se requiere.

\section{Referencias bibliográficas}

Barbosa, L. (2004). Sociedade de consumo. Río de Janeiro: Jorge Zahar.

Baudrillard, J. (2009). La sociedad de consumo: sus mitos, sus estructuras. Madrid: Siglo XXI.

Bauman, Z. (2015). Modernidad líquida. Buenos Aires: Fundación de Cultura Económica.

Calenge, B. (2003). Publics nomades, bibliothèque familière: enquêtes sur le public de la bibliothèque municipale de Lyon. Bulletin des Bibliothèques de France (BBF), 48(6), pp. 66-72. Recuperado de http://bbf.enssib.fr/consulter/bbf-2003-06-0067-001

De Rosa, C., Dempsey, L., y Wilson, A. (2004). The 2003 ocLC environmental scan: pattern recognition: executive summary. Recuperado de http://oclc.org/content/dam/oclc/reports/escan/downloads/escansummary en.pdf

De Rosa, C., Cantrell, J., Cellentani, D., Hawk, J., Jenkins, L., y Wilson, A. (2005). Perceptions of libraries and information resources: a report to the oCLC membership. Recuperado de http://www.oclc.org/content/dam/oclc/reports/pdfs/Percept_all.pdf

De Rosa, C., Cantrell, J., Carlson, M., Gallagher, P., Hawk, J., y Sturtz, C. (2011). Perceptions of libraries, 2010: context and community. A report to the oCLC membership. Recuperado de http://www.oclc.org/content/dam/oclc/reports/2010perceptions/2010perce ptions_all.pdf

Domènech, M., Tirado, F. J., Traveset, S., y Vitores, A. (1999). La desinstitucionalización y la crisis de las instituciones. Educación Social, (12), pp. 20-32. Recuperado de https://www.raco.cat/index.php/EducacioSocial/article/download/144294/ $\underline{383946}$

Dominzain, S., Radakovich, R., Duarte, D., y Castelli Rodríguez, L. (2014). Imaginarios y consumo cultural: tercer informe nacional sobre consumo y comportamiento cultural. Recuperado de http://dspace.mides.gub.uy:8080/xmlui/handle/123456789/599 
García Canclini, N. (2008). Libros, pantallas y audiencias: ¿qué está cambiando? Comunicar, 15(30), pp. 27-32. https://doi.org/10.3916/c30-2008-01-004

García Canclini, N., Gerber Bicecci, V., López Ojeda, A., Nivón Bolán, E., Pérez Camacho, C., Pinochet Cobos, C., y Winocur Iparraguirre, R. (2015). Hacia una antropología de los lectores. Recuperado de https://www.fundaciontelefonica.com/cultura-digital/publicaciones/469/

García Pérez, J. F. (2011). Propiedad intelectual: la información como bien público y bien privado. En E. Morales Campos. (Coord.), Derecho a la información, bien público y bien privado: acceso comunitario y acceso individual (pp. 81-95). Recuperado de http://files.sld.cu/bmn/files/2014/07/super-derecho_a_la_informacion.pdf

García Rivadulla, S. (2013). Percepción 2.0: las bibliotecas universitarias uruguayas en la web social desde el punto de vista del usuario (Tesis de maestría, Universidad Carlos III de Madrid. Departamento de Biblioteconomía y Documentación). Universidad Carlos III de Madrid, Getafe. Recuperado de https://core.ac.uk/download/pdf/16292464.pdf

González Carballal, S. M. (2019). La biblioteca en la sociedad de consumo: en un estudio de casos en la Universidad de la República (Tesis de maestría inédita, Universidad de la República. Facultad de Información y Comunicación). Universidad de la República. Facultad de Información y Comunicación, Montevideo.

Las bibliotecas japonesas son también cafeterías, gimnasios y salones de belleza. (1 de julio de 2017). Infobae. Recuperado de https://www.infobae.com/america/wapo/2017/07/01/las-bibliotecasjaponesas-se-convierten-en-cafeterias-gimnasios-y-salones-de-belleza/

Lipovetsky, G., y Serroy, J. (2015). La estetización del mundo: vivir en la época del capitalismo artístico. Barcelona: Anagrama.

Mattelart, A., y Piemme, J. M. (1982). Las industrias culturales: génesis de una idea. En A. Anverre et al., Industrias culturales: el futuro de la cultura en juego (pp. 62-79). Recuperado de http://unesdoc.unesco.org/images/0013/001353/135399so.pdf

Pignet, D. (2005). La bibliothèque peut-elle survivre à ses consommateurs? Bulletin des bibliothèques de France (BBF), 50(1), pp. 38-45. Recuperado de http://bbf.enssib.fr/consulter/09-peignet.pdf

Radakovich, R. (2011). Retrato cultural: Montevideo entre cumbias, tambores y óperas. Recuperado de 
http://archivos.liccom.edu.uy/Libro\%202\%20Retrato\%20cultural\%20defi nitivo.pdf

Sassatelli, R. (2012). Consumo, cultura y sociedad. Buenos Aires: Amorrortu.

Seibel, B. (2005). Bibliotheques, librairies, grandes surfaces et vente par correspondance, quelles concurrences autour du livre? Publics: quelles attentes? Bibliotheques: quelles concurrences? (pp. 43-50). París: Éditions de la Bibliotheque publique d'information.

Stolovich, L. (2001). La lógica económica del empleo cultural. En La cooperación multilateral en red para la cultura del nuevo milenio: actas del 2. ${ }^{\circ}$ Campus Euroamericano de Cooperación Cultural (Cartagena de Indias, Colombia, 10-14 dic, 2001). Recuperado de http://www.oei.es/historico/euroamericano/ponencias_mercado_logica.php

Torres Santo Domingo, M. (2005). La función social de las bibliotecas universitarias. Boletín de La Asociación Andaluza de Bibliotecarios, (80), pp. 43-70. Recuperado de https://www.redalyc.org/pdf/353/35308004.pdf

\section{Notas}

${ }^{1}$ Los antecedentes encontrados fueron: los mencionados de la OCLC (De Rosa, Dempsey y Wilson, 2004; De Rosa et al., 2005, 2011); la tesis realizada por Sandra García Rivadulla (2013), que estudia la percepción de las bibliotecas universitarias uruguayas en el contexto de la web social; y los informes de Dominzain, Radakovich, Duarte y Castelli Rodríguez (2014) y Radakovich (2011), respecto a la relación de las personas con la lectura.

Nota: El editor Mario Barité aprobó este artículo.

Nota de contribución: La totalidad del trabajo estuvo a cargo de González Carballal.

Corrección de estilo realizada por Raquel Souto en el marco del convenio entre la Tecnicatura universitaria en corrección de estilo (FHCE) y la Facultad de Información y Comunicación (Udelar). 